\section{Gene mapping at the crossroads}

\author{
by Mary Lindley
}

THE human gene map is said to be poised for rapid expansion, during the next few years, as the techniques of molecular biology and immunology take firm root in this traditional domain of geneticists and cell biologists. At a recent reunion in Edinburgh*, 150 of the aficionados agreed that they have now identified the positions of about 350 genes on the human chromosomes. Although the current rate of increase is a mere two or three genes per month, the atmosphere was full of confidence that this will accelerate rapidly, making a complete human gene map a possibility within the lifetime of some of the younger members of the gene mapping fraternity.

One of the most promising strategies involves the combination of some of the familiar tools of cytogenetics with techniques developed to investigate the fine structure of both prokaryotic and eukaryotic genes. By such methods the B-globin gene, already believed to be on chromosome 11 , has been localised more precisely by D. Housman (Massachusetts Institute of Technology) and colleagues. They used cells from hybrid clones each of which contained the Chinese hamster genome together with a section of human chromosome 11 . To identify the section carrying the $\beta$-globin gene they extracted the DNA from cells of each clone, treated it with a restriction endonuclease and separated the resulting fragments on agarose gel. The separated fragments were transferred onto nitrocellulose filters and hybridised to a radioactively labelled cloned human $\beta$-globin DNA probe. The pattern of hybridisation revealed by autoradiography showed that the gene for human B-globin is carried on a section of the short arm of chromosome 11. Because the gene for $\beta$-globin is known to be linked to those for $\gamma, \delta$, and $\varepsilon$ globins, all four genes must be carried on this chromosomal region.

By a similar procedure, B.R. Migeon and colleagues (Johns Hopkins University School of Medicine, Baltimore) have used hybrid cells containing the mouse genome and a human chromosome resulting from a translocation between chromosome 11 and the $\mathrm{X}$ chromosome (Proc. natn. Acad. Sci. U.S.A. in the press). They were able to select for the presence or absence of the human chromosome because it carried a functioning gene for the enzyme hypoxanthine guanine phosphoribosyl transferase, which the mouse cells lacked. These experiments have further confirmed that the $\beta$ and $\delta$-globin genes are carried on human chromosome 11 , and show that they are not on the segment involved in the

"The fifth International Workshop on Human Gene Mapping was held in Edinburgh on 9-13 July 1979. It was organised by H.J. Evans, MRC Clinical and Population Cytogenetics Unit, Edinburgh. The abstracts and committee reports will be published in Cytogenetics and Cell Genetics. translocation.

The application of 'restriction mapping' to increasing numbers of genes now depends on the rate at which cloned probes can be producd and characterised. F.H. Ruddle (Yale University) predicted that restriction mapping will become the predominant method for mapping genes, and that the major part of the human gene map could be known by the end of this century.

Speed, convenience and scope for fine resolution should give restriction mapping an advantage over in situ hybridisation, which has recently revealed further information about the arrangement of the histone genes on chromosome 7. Using labelled probes of complementary RNA, prepared from cloned sea urchin genes, D.M. Steffensen (University of Illinois, Urbana) and J.J. Yunis and M.E. Chandler (University of Minnesota Medical School) have both localised the five human histone genes to the long arm of this chromosome. Their results, however, do not indicate precisely the same positions, with the latter group placing the cluster of genes nearer to the end of the long arm (Chandler et al. Science, in the press). The difference may be due to the difficulty of interpreting the pattern of silver grains in the final, autoradiographic stage of this procedure.

Another promising line of enquiry for the future is provided by cell surface molecules which can be recognised by biochemical and immunological techniques and correlated with the presence of particular chromosomes. A. de la Chapelle (Folkhalser Institute of Genetics, Helsinki) and colleagues have followed up previous studies which indicated an association between monosomy for chromosome 7 and defective granulocyte chemotaxis (Rutuu et al. Nature 265, 146; 1977). Treating the defective monosomic granuloctyes with a radioactive label specific for glycoproteins, the Helsinki group has shown that these blood cells have much less than the normal amount of one particular glycoprotein. This suggests that a gene on chromosome 7 codes for a glycoprotein associated with chemotaxis. The same chromosome may also be involved in the expression of receptors for epidermal growth factor, according to N. Shimizu and colleagues (University of Arizona, Tucson). They have correlated the presence of this chromosome in hybrid cells with the ability to bind epidermal growth factor.

Indirect immunofluorescence, used by C.K. Eun and H.P. Klinger (Albert Einstein College of Medicine, New York), has suggested that the expression of fibronectin on cell surfaces is affected by chromosome 11. This contrasts with the suggestion of Owerbach et al. (Proc, natn. Acad. Sci. U.S.A. 75, 5640; 1978), who labelled surface proteins by lactoperoxidase-catalysed iodination and correlated the presence of fibronectin with chromosome 8. The disagreement arises again in the results of a similar study by $\mathrm{M}$. Smith (Mount Sinai School of Medicine, New York) and colleagues who found that hybrid cells could be positive for fibronectin while lacking glutathione reductase which is associated with chromosome 8 . The genetics of fibronectin it seems may be complex, and possibly may involve genes on various chromosomes. On the other hand, the discrepancy may be resolved by the application of more refined techniques.

Another promising area is represented by two reports of genes that influence the expression of oncornaviruses. Using human-mouse cell hybrids, C.E. Wright and T.B. Shows (Roswell Park Memorial Institute, Buffalo) have found a gene on human chromosome 11 that induces the expression of a mouse xenotropic oncornavirus and another on chromosome 15 involved in the induction of endogenous mouse $\mathrm{N}$-tropic oncornavirus. They call these genes $B V I X$ and $B V I N$, respectively, and have found that they are carried on human chromosomes homologous to mouse chromosome regions that also carry genes affecting the expression of oncornaviruses.

This suggestion of genetic conservation during evolution is just one of many examples that are emerging as gene maps are developed for different mammalian species. As well as those of mouse, rabbit and various primates, genes are now being assigned to the chromosomes of cow, sheep, pig, cat, dog and horse. As would be expected, these are providing further evidence of the conservation of the $X$ chromosome during evolution. It is also becoming increasingly clear that other linkage groups have been strongly conserved, especially between the great apes and man. Yunis and Chandler, for example, have extended their in situ hybridisation study to chimpanzee, gorilla and orangutan and localised the histone genes on homologous regions of the counterparts of human chromosome 7.

Similarly J.J. Garver (State University, Leiden) and colleagues have localised the major histocompatibility complexes of chimpanzee and rhesus monkey on a chromosome homologous with human chromosome 6, which carries the HLA complex. P.A. Lalley and colleagues (Oak Ridge National Laboratory) have found that some genes on human chromosomes 1 and 6 are correspondingly linked in the baboon, indicating conservation. On the other hand, they interpret their study of the baboon counterpart of human chromosome 2 , as a sign that extensive chromosomal rearrangements have occurred during the evolution of this chromosome. Many more such insights should come as the various gene maps develop.

Mary Lindley is an Assistant Editor of Nature. 